\title{
Laser-induced graphitization of a forest-based ink for use in flexible and printed electronics
}

\author{
Jesper Edberg $\mathbb{D}^{1 凶}$, Robert Brooke ${ }^{1}$, Omid Hosseinaei ${ }^{2}$, Andreas Fall ${ }^{2}$, Kosala Wijeratne ${ }^{1}$ and Mats Sandberg (iD ${ }^{1}$
}

Laser-induced graphitization (LIG) is a method of converting a carbon-rich precursor into a highly conductive graphite-like carbon by laser scribing. This method has shown great promise as a versatile and low-cost patterning technique. Here we show for the first time how an ink based on cellulose and lignin can be patterned using screen printing followed by laser graphitization. Screen printing is one of the most commonly used manufacturing techniques of printed electronics, making this approach compatible with existing processing of various devices. The use of forest-based materials opens the possibility of producing green and sustainable electronics. Pre-patterning of the ink enables carbon patterns without residual precursor between the patterns. We investigated the effect of the ink composition, laser parameters, and additives on the conductivity and structure of the resulting carbon and could achieve low sheet resistance of $3.8 \Omega \mathrm{sq}^{-1}$ and a high degree of graphitization. We demonstrated that the process is compatible with printed electronics and finally manufactured a humidity sensor which uses lignin as the sensing layer and graphitized lignin as the electrodes.

npj Flexible Electronics (2020)4:17; https://doi.org/10.1038/s41528-020-0080-2

\section{INTRODUCTION}

Carbon, one of the most abundant elements, in not only the foundation of all life but also an integral part in our society. Due to its four valence electrons, carbon easily forms bonds with other elements, which is the basis for all organic molecules, both synthetic and natural. Carbon also bonds with itself and comes in various allotropes such as amorphous carbon, graphite, diamond, fullerenes, carbon nanotubes, and graphene. Due to its mechanical, electrical, and electrochemical properties, carbon appears in many products, all from building materials ${ }^{1}$ to nanosized electronics circuits ${ }^{2}$. The discovery of graphene, with its ballistic charge transport ${ }^{3}$ and mechanical strength 100 times greater than steel $^{4}$, has sparked a new carbon revolution.

Carbon has been used for a long time in various electrochemical devise and processes due to its chemical/electrochemical resistance. In lithium ion batteries, carbon makes up the porous negative electrode (anode) where the lithium ions are intercalated to store energy ${ }^{5-8}$. Likewise, most commercial supercapacitors are based on combinations of conductive carbon (such as carbon black) and high specific surface area activated carbon ${ }^{9}$. Nanomaterials such as graphene and carbon nanotubes have the potential to further improve such devices, yielding higher energy, power densities, and mechanical rigidity ${ }^{10,11}$.

In the field of printed electronics, where electronic and electrochemical devices are produced by patterning functionalized inks using various printing methods, carbon is often used to make conductors, protective layers, and electrodes ${ }^{12,13}$. Printed electronics has the potential of large area and high-throughput manufacturing on flexible substrates such as plastic or paper, which will be necessary to produce low-cost devices in bulk for internet-of-things (IoT), biomedical, and wearable electronics applications ${ }^{14}$. Such devices will likely largely be disposable, which means that they will eventually end up in the garbage bin to be sent to landfills or to be burned, or in the worst-case scenario ending up in our forests and oceans. For this reason, there is a growing interest to make printed electronics from materials that are environmentally friendly with respect to their origin, their processing, and their disposal. Most commercial carbon printing inks (and carbon products in general) are derived either from petroleum or mining. A greener alternative would be to use carbon derived from biomass such as wood which can be carbonized/graphitized to become conductive. Not only is this source of carbon part of the natural $\mathrm{CO}_{2}$-cycle, but there is also vast amounts of unused by-products from the biorefinery and pulp and paper industries.

Recently, a new method of converting a carbon-rich source into conductive carbon was invented. The technique which is called "laser-induced graphitization" (LIG) uses a laser for the graphitization as opposed to an oven, which is often used for this process ${ }^{15}$. The carbon source, which is typically a polymer, can be of either synthetic or natural origin. Not only can the process be run at a relatively low power, it also opens the possibility of patterning the carbon by using a system such as a laser engraver. It has been shown that this graphitization technique can be used to produce graphene and therefore it has the potential for producing highly conductive materials for use in various applications like energy storage devices $^{16-19}$, electrodes for hydrogen evolution ${ }^{20}$, biomedical application ${ }^{21}$, and sensors ${ }^{22}$. In general, lignocellulosederived carbonaceous materials are considered as nongraphitizable carbons ${ }^{23}$. However, it has been shown that LIG is an efficient method to rapidly reach a relatively high degree of graphitization in lignocellulosic materials ${ }^{24}$. Several bio-based carbon sources like wood and paper have been used in $\mathrm{LIG}^{24-26}$. For bio-sources, which contains lignin, it's been determined that there is a correlation between the amount of lignin and the graphite formation such that LIG is favored by a high-lignin content ${ }^{25}$. It has therefore been suggested that the cross-linked aromatic structure of lignin is important in this process, making wood a good precursor material ${ }^{27}$. However, wood has little practical use in electronics due to the limits in formation of different geometries such as films. Instead, by making a coating of

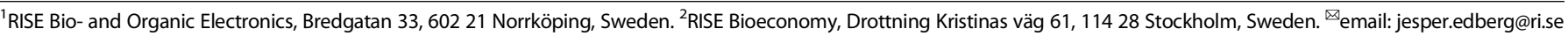


wood-derived component on a flexible substrate it could be combined with printed electronics.

Herein, we demonstrate how screen printing, one of the most commonly used printing methods in printed electronics, can be combined with LIG to make electronic circuits and devices on flexible substrates. This is achieved by making an ink based on chemically modified cellulose and lignin, which is screen printed and subsequently graphitized using a commercial $\mathrm{CO}_{2}$ laser engraver. We investigate how the ink composition and laser power affects the electrical performance of the produced graphite and how this is related to its microstructure. We further demonstrate how printing in combination with laser patterning is compatible with printed electronics and show how it can be used to make resistors where the resistance is controlled by the laser power rather than the resistor form factor. Finally, we show how both the ink and the graphitized ink can be used to make humidity sensors with excellent response using a single printing step.

\section{RESULTS AND DISCUSSION}

\section{Lignographic patterning by screen printing}

Although LIG has been demonstrated on bio-based materials such as wood, it has limited applications due to the bulky and rigid nature of this material. By deconstructing wood into its main components, lignin and cellulose, it is possible to formulate a printing ink that can be applied to various substrates, including flexible ones like PET. Besides the choice of substrate and thickness of the coated wood material, this approach also gives the opportunity of tuning the ratio of cellulose, lignin, and other additives, something which is not possible in unprocessed wood. Zhang et al. ${ }^{26}$ prepared a printable ink based on lignin and polyvinyl alcohol (PVA) and produced thin films by blade coating followed by laser scribing to produce conductive patterns. Although this technique showed impressive conductivity, its usefulness is limited by the need to dissolve the remaining lignin ink after the graphitization step, which is both time consuming and adds additional processing steps. The carbon patterning has little usefulness in and by itself but could have many applications when combined with printed electronics. In order to make LIG compatible with printed electronics, we developed a wood-based ink that can be pre-patterned by screen printing followed by laser graphitization. In this way, there is no residual ink between the carbon patterns (which would otherwise create ionic shorts and thereby capacitive effects), and so additional printing layers can immediately be applied to produce various devices and circuits.

To be fully forest-based and environmentally friendly, we used cellulose (2-HEC) as the binder for the ink and water as the solvent. 2-HEC is commonly used as a binder and gelling agent in ink preparation, as well as cosmetic, pharmaceutical and food products. We refer to the ink as "wood-ink" and the whole process as "lignography". Figure 1a shows a schematic of the lignography process, as well as the molecular structures of the lignosulfonate and 2-HEC used in the ink. The ink is printed onto flexible polyethylene terephthalate (PET) sheets and quickly dries, either in air or in oven for accelerated drying. A commercial $\mathrm{CO}_{2}$ laser was used to convert the ink into graphite. The insets of Fig. 1a shows photographs of carbon patterns on a global print of the wood ink on PET as well as a carbon pattern without residual ink between the patterns produced on a cardboard box. Figure $1 \mathrm{~b}$ shows a photograph of several wood ink patterns, where one of the patterns has further been graphitized. The ink and carbon patterns do not crack when bending the substrate (see "Integrating lignography with printed electronics" section). Inks with different lignin:cellulose $(L: C)$ weight ratio was prepared and tested for printability. Figure 1c shows printed lines of an ink with $L: C$ 1:2 ratio and Fig. 1d with $L: C$ 2:1 ratio, before and after LIG. It is evident from the photographs that the ink with higher lignin content has a more homogenous pattern. This ink was also less prone to dry out in the screen-printing frame.

In addition to the advantage of not having residual ink between the carbon patterns, pre-patterning of the lignin ink also has an impact on the patterning resolution. As has been previously reported ${ }^{25}$, to obtain the best graphitization results the laser is kept at certain defocus ( $3 \mathrm{~mm}$ in this work). This has an impact on the laser spot size which will be considerably enlarged and sets a limit to how small patterns can be produced on global layers of the wood ink. This is shown in Supplementary Fig. 1 where different patterns were produced on global prints. As can be seen, the lines blend together for the smaller patterns due to the large laser spot size. However, by pre-patterning the lignin ink to smaller dimensions than the laser spot size, this limitation can be circumvented. Although the laser will touch the PET outside the lignin pattern, only the ink will carbonize since the laser has no effect (except for some light etching) on the substrate. The resolution limit then becomes limited by the printing resolution, which depends on several factors such as the printing technique, ink viscosity, and substrate surface energy. Each printing technique possesses advantages and disadvantages. For example, inkjet printing can produce high resolution patterns $(30-70 \mu \mathrm{m})$ but suffers from slow printing speed $\left(0.001-0.1 \mathrm{~m} \mathrm{~s}^{-1}\right)^{28}$. Transfer printing is another technique, which offers high resolution, but is more suited for producing thin films ${ }^{29,30}$. Screen printing was chosen for this work because of a good trade-off between resolution $(50-100 \mu \mathrm{m})$, printing speed $\left(0.1-1 \mathrm{~m} \mathrm{~s}^{-1}\right)$, and produced film thickness $(5-100 \mu \mathrm{m})^{28}$.

If one prefers to produce the patterns on global layers followed by dissolving the lignin ink, this is also possible. Supplementary Fig. 2 shows how the residual ink in the pattern shown in Fig. 1a has been dissolved by water. The carbon pattern, however, is not likely to dissolve in water as the carbonization have made it hydrophobic and chemically fused (i.e., cross-linked) the pattern. In addition, it seems like the carbon pattern has good enough adhesion to the substrate to stay on it even after being submerged for extended periods of time.

To make truly environmentally friendly electronics, also the substrate should be bio-based. The inset of Fig. 1a as well as Supplementary Fig. 3 show photographs of lignography performed on cardboard with glossy or unprocessed surfaces respectively. However, due to the rough surface structure and wetting issues of cardboard and paper, reproducibility was poor. As the focus of this work was on the printing process and the ink, synthetic thermoplastic substrates were used for the bulk of the work which is the standard in printed electronics at the moment. Future work will focus on applying the lignography process on bio-based substrates.

\section{Electrical performance}

The electrical performance of the obtained carbon patterns was evaluated by four probe resistance measurements to obtain the sheet resistance. Squares $(40 \times 40 \mathrm{~mm})$ of lignin ink was printed on PET sheets and smaller squares $(15 \times 15 \mathrm{~mm})$ were subsequently graphitized as shown in Fig. 2a. Each data point in the electrical measurements represents one measurement on one carbon square. Sheet resistance was evaluated for various laser power and ink composition. The variation in ink composition includes the lignin:cellulose $(\mathrm{L}: \mathrm{C})$ weight ratio and the addition of boric acid (BA) to the ink. Boric acid is a flame retardant which has previously been used in LIG to perform the graphitization on carbon sources like cellulose in ambient atmosphere ${ }^{25,31}$. Indeed, most demonstrations of LIG were performed in inert atmosphere to prevent combustion and maximize the degree of graphitization. Figure $2 b$ shows how the sheet resistance varies with laser power and $L: C$ ratio. Figures $2 c$, d further show more clearly the sheet resistance 

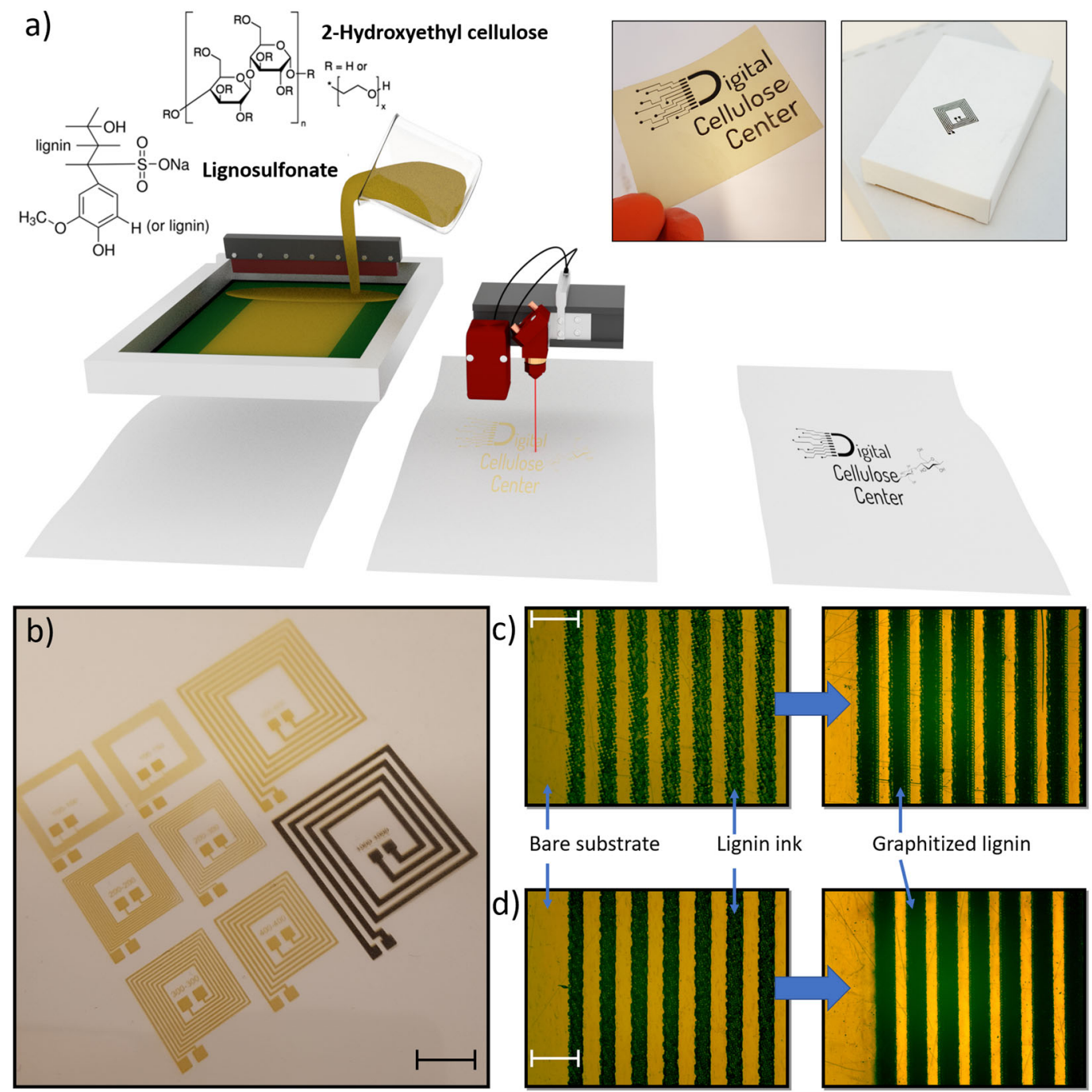

Fig. 1 Overview of the lignographic technique. a Molecular structures of the wood ink components as well as the printing and lasing procedure with insets showing photographs of patterns graphitized on global prints on flexible substrates and on cardboard. $\mathbf{b}$ Photograph of screen-printed patterns of wood ink where one of the patterns has been graphitized. Scale bar $1 \mathrm{~cm}$. c Printed lines ( $300 \mu \mathrm{m}$ width) of an ink with high cellulose content (L:C 1:2) before (left) and after (right) graphitization. Scale bar $1 \mathrm{~mm}$. d Printed lines of an ink with high-lignin content (L:C 2:1) before (left) and after (right) graphitization. Scale bar $1 \mathrm{~mm}$. It should be noted that while the background in c and d resemble the color of the wood ink, this is actually the substrate colored by the light of the microscope.

at high and low laser power, respectively. As is evident from the graphs, sheet resistance decreases with increasing laser power. This behavior was expected as it has previously been reported ${ }^{15,24,26}$. What was more surprising was the sharp increase in resistance when the amount of cellulose reaches a certain point. As cellulose by itself does not carbonize in ambient atmosphere and is instead etched away, we expected a gradual increase in resistance with increasing amount of cellulose. Instead, even with twice as much cellulose as lignin, low values of sheet resistance could still be obtained, which is comparable or even lower than the samples with higher lignin loading. The sharp increase in resistance at [ $L: C$ 1:3] indicates a percolation threshold where the lignin no longer forms a well-connected network or due to the formation of smaller crystallites (see discussion in "Micro- and macrostructure" section).

Even though the prints would graphitize in ambient atmosphere even with high loading of cellulose, we wanted to investigate if the addition of boric acid could further improve the graphitization by suppressing combustion. The results are shown in Fig. 2e, f, where prints with L:C 2:1 ratio were graphitized at low and high laser power with different amounts of BA added to the ink. This particular $\mathrm{L}: \mathrm{C}$ ratio was used as it displayed the best overall performance when also taking into account the printability and drying time of the ink. The addition of BA resulted in a reduction in sheet resistance with the best performance being $\sim 6 \Omega \mathrm{sq}^{-1}$. at high laser power. Besides laser power there are many other processing parameters that affect the sheet resistance, and not all could be investigated in-depth in this work. This includes the laser speed, defocus, and number of passes ${ }^{25}$. However, by trial and error the best sheet resistance reached was $3.8 \pm 0.1 \Omega \mathrm{sq}^{-1}$, which was obtained at two laser passes at high power (11\%) and with boric acid (L:BA 10:1). To the best of our knowledge, this is the lowest value of sheet resistance reported for LIG using bio-based sources.

Like most previous work on LIG, we have focused on sheet resistance rather than conductivity ${ }^{15,24,25}$. Even though conductivity is an important parameter from a materials investigation point of view, sheet resistance is the most important parameter when considering the usefulness of the technology. Conductivity is a useful value when there are no limitations on the dimensions 
a)
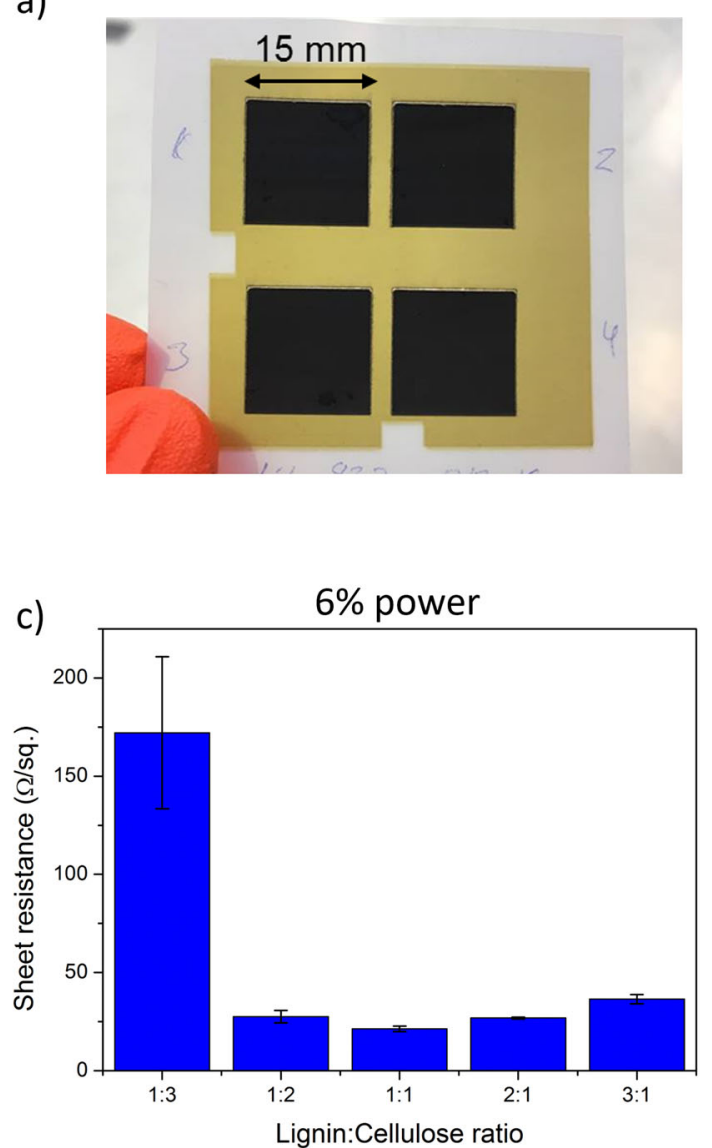

e)

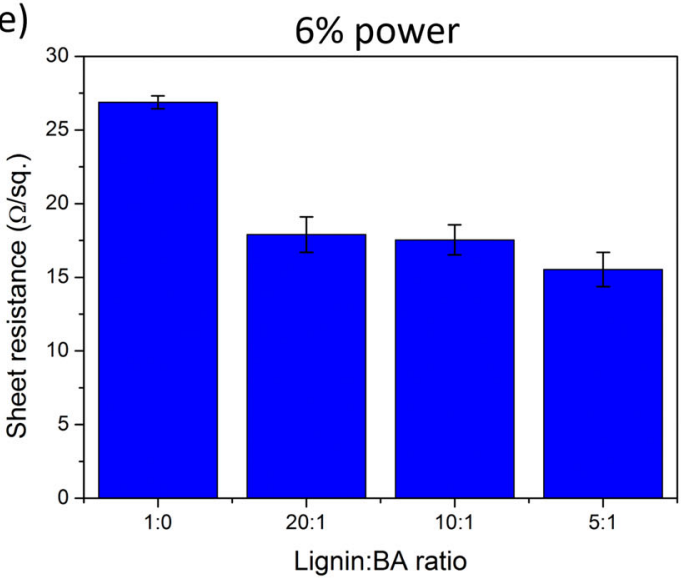

b)

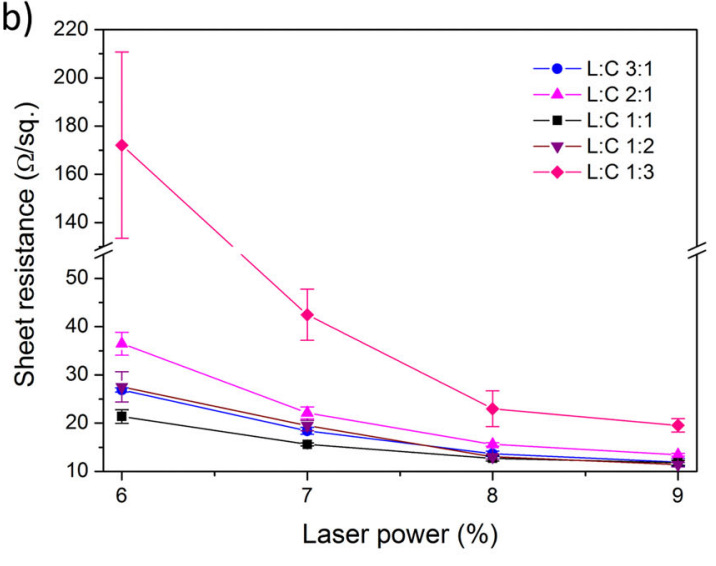

d)

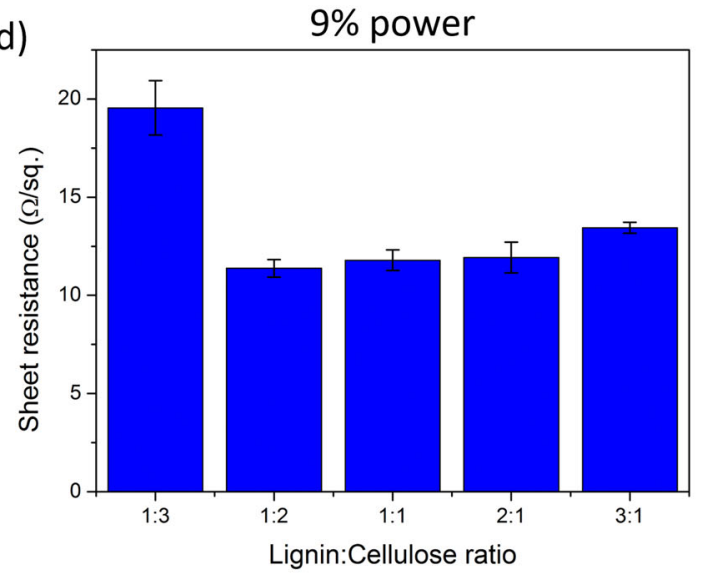

f)

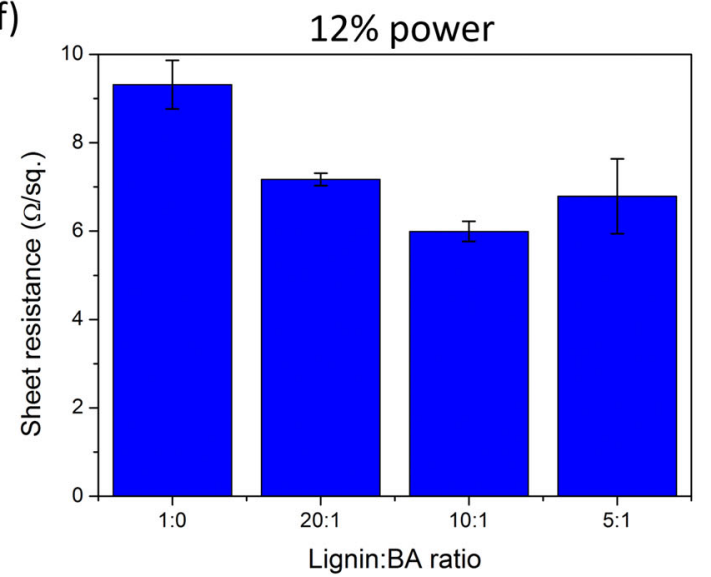

Fig. 2 Electrical characterization. a Photograph of the lased squares used to measure sheet resistance, b sheet resistance vs. laser power for different lignin:cellulose $(\mathrm{L}: \mathrm{C})$ ratios, $\mathbf{c}$, $\mathbf{d}$ sheet resistance for different $\mathrm{L}: \mathrm{C}$ ratios at $6 \%$ and $9 \%$ laser power, respectively, e, $\mathbf{f}$ sheet resistance for different lignin:BA ratios at $6 \%$ and $12 \%$ laser power, respectively, using L:C 2:1 ratio. c-f Mean values and SD.

on the conductor such that the resistance can be decreased, e.g., by increasing the thickness of a conductive film. However, in the case of lignography, there is a limit to the laser penetration depth and therefore to the maximum thickness making it a 2Dtechnology where sheet resistance becomes a better comparison to other similar technologies. That being said, the conductivity of different samples was calculated by measuring the thickness of the graphitized films using cross section SEM imaging (Supplementary Fig. 4). The best obtained conductivity was $\sim 28 \mathrm{~S} \mathrm{~cm}^{-1}$, which is in the same order of magnitude as previous reports of LIG using similar materials ${ }^{26}$. Although the prints had a thickness in the order $8-15 \mu \mathrm{m}$, the thickness of the carbon was considerably thicker, sometimes $>100 \mu \mathrm{m}$. This indicates that the material "inflates" upon graphitization and becomes more porous. This is also shown in SEM imaging which is further discussed in "Microand macrostructure" section. The induced porosity and volume expansion are likely due to the partial combustion and volatilization of the cellulose and/or lignin. By applying the Reynolds and Hough rule, we can get an estimate of the intrinsic conductivity of the carbon by normalizing the conductivity with the volume fraction of carbon (i.e., by excluding the air-filled pores) ${ }^{32}$. This method has previously been used to estimate the intrinsic 
conductivity of biochar carbon in supercapacitors ${ }^{33}$. As we know the original thickness of the ink then we can easily estimate the upper limit of the carbon volume fraction in the graphitized samples. Applying the Reynolds and Hough rule give us values between 112 and $210 \mathrm{~S} \mathrm{~cm}^{-1}$ of the intrinsic conductivity, which is similar to values reported for lignin graphitized in inert environment in an oven (Shao et al. ${ }^{34}$ reported $95 \mathrm{~S} \mathrm{~cm}^{-1}$ after pyrolysis at $1000^{\circ} \mathrm{C}$ ). Even without applying the Reynolds and Hough rule, the conductivity and sheet resistance of the lignographic carbon is in the same order or better than most reported graphene-based inks used for printed and flexible electronics ${ }^{35}$. It should be noted that such graphene-based inks often use hazardous organic solvents like NMP or DMF and require high temperature annealing $\left(200-400{ }^{\circ} \mathrm{C}\right)$ for more than $30 \mathrm{~min}$ in order to reach these conductivity values, which is more than what most flexible substrates used in printed electronics can handle and also a time consuming process. If the volume expansion can be suppressed through further optimization of the ink formulation and lignography process, even higher values might be possible to reach by creating a densified and more interconnected carbon network.

\section{Micro- and macrostructure}

In order to understand what is happening to the material when using different inks and laser power we investigated the microand macrostructure of the obtained carbon using wide-angle X-ray scattering (WAXS) and Scanning Electron Microscopy (SEM). The WAXS measurements provides information about the crystal structure of carbon and the degree of graphitization. Supplementary Table 1 and Supplementary Fig. 5 give all crystallographic data for different samples. This includes the $d$-spacing $\left(d_{002}\right.$ and $\left.d_{100}\right)$ as well as the crystallite sizes which were reported as out-of-plane (stack height, $L_{c}$ ) and in-plane (stack size, $L_{a}$ ). The interplanar spacing $\left(d_{002}\right)$ gives an indication of the degree of graphitization during the LIG process. Smaller $d$-spacing is associated with higher degree of graphitization. As graphite is more conductive than amorphous carbon, it is desirable to obtain a small $d$-spacing. A "perfect" graphite stack will have an interlay spacing $\left(d_{002}\right)$ of $3.34 \AA^{36}$. As can be seen from Supplementary Table 1, the $d$ spacing $\left(d_{002}\right)$ in the graphitized samples was $3.39-3.41 \AA$. Although the sample with the highest cellulose content had a slightly lower $d$-spacing, in general different formulations and lignography parameters did not significantly change the $d$-spacing values. This can indicate effectiveness of LIG in formation of graphitic structure in different formulations. Further investigation is needed to evaluate the effect of LIG parameters or formulation of ink on properties such as porosity, elemental composition and carbon yield. The carbon produced in this work has a smaller $d$ spacing and hence a higher degree of graphitization compared to the most of lignin and/or cellulose samples carbonized in furnace. The data reported on $d$-spacing of lignin or cellulose carbonized in furnace at $1000{ }^{\circ} \mathrm{C}$ are usually in the range of $4.05-3.75 \AA^{34,37-40}$. This value can reduce to the range of 3.64 to $3.40 \AA$ when carbonization temperature significantly increases $(2000$ to $\left.2700^{\circ} \mathrm{C}\right)^{37,41-44}$. The reported crystallite sizes (both $L_{c}$ and $L_{a}$ ) are higher than most of data reported on crystallite size of lignin and/ or cellulose carbonized in furnace even at the elevated temperature (up to $\left.2700^{\circ} \mathrm{C}\right)^{34,37,38,41-44}$. The presented results on crystallography of the current samples prepared by LIG are interesting since graphitization typically require temperatures higher than $2500^{\circ} \mathrm{C}$ in inert atmosphere while the carbon in this work was produced in presence of oxygen using a low power laser.

Figure 3 shows the crystallite size for samples produced with different $\mathrm{L}: \mathrm{C}$ ratios, laser power, and amount of boric acid. The crystallite size (especially the stack size) correlates well with the sheet resistance from Fig. 2 such that larger crystallites give lower sheet resistance, i.e., larger amount of lignin, higher laser power, and higher BA loading all resulted in larger crystallites and also lower sheet resistance. Although the L:C 1:3 sample had the smallest interplane spacing, which would suggest a larger degree of graphite, the high sheet resistance in this sample might be explained by the small crystallite size. Figure $3 \mathrm{~d}$ shows a comparison between the crystallite size as well as the interplane spacing of one of the best lignographic samples and published data on lignin- and cellulose-based carbon ${ }^{34,41,44}$. Although crystallite size was larger and $d$-spacing smaller in this work compared to, e.g., the lignin/cellulose comparison, the conductivity was still smaller in this work $\left(\sim 30 \mathrm{~S} \mathrm{~cm}^{-1}\right.$ compared to $\sim 100 \mathrm{~S} \mathrm{~cm}^{-1}$ ). This is counterintuitive since large crystallites and small $d$-spacing should be advantageous for charge transport. An explanation to this can be found in Fig. 4, which shows SEM images of graphitized samples. The microscopy images show a large degree of porosity and a foam-like structure in all samples which may explain the comparatively low conductivity as was also discussed in "Electrical performance" section. This suggests that while the global conductivity which takes air pores into account is lower compared to literature, the microscopic conductivity might be better. It appears that the samples with the lower sheet resistance also have more compact structure, for example, when comparing Fig. 2b, c, where Fig. 2c was produced with higher power, or Fig. 2c, e, where Fig. 2e contained BA. Although a denser structure is beneficial for lower sheet resistance, an open porous structure can be advantageous for certain applications such as when used as the electrodes in supercapacitors, batteries or in electrochemical processes ${ }^{15,20}$.

\section{Integrating lignography with printed electronics}

Although many previous publications on LIG have shown standalone applications such as conductor tracks or as electrodes, demonstrations of integration have been sparser. To this end, we used lignography together with commercial screen-printing inks to show the compatibility with printed electronics. Figure 5a shows a schematic of a carbon resistor with printed silver contacts and a protective lacquer layer, and Fig. $5 \mathrm{~b}$ shows the resistance of such resistors produced using different laser power. The lignin ink was first screen-printed into a matrix of equally sized rectangles followed by graphitization at different laser powers (Fig. 5c). This was followed by the screen-printing of the silver contacts (Fig. $5 \mathrm{~d}$ ) and the lacquer layer (Fig. 5e). Although this is a simple design, it demonstrates the possibility of printing on top of the lignographic carbon which is necessary for manufacturing more complex circuits and devices. The resistors also demonstrate an advantage over conventional screen-printed carbon in that the resistance values can be modulated without changing the form factor of the resistors. Normally, the sheet resistance of such screen-printed carbon can only be modulated by multiple prints, which is time consuming and might require multiple frames if a resistor network with different resistor values are required. Alternatively, the width or length of the carbon patterns can be modified, but this also has its limitations since large resistance values will require long patterns which takes up space. By using lignography, the resistor values could be modulated over 5 orders of magnitude without changing the form factor. Supplementary Table 2 shows the resistance and device yield both after the silver print and the lacquer print. A high yield was kept for laser power up to $6 \%$ but dropped dramatically at higher power. The drop in yield was due to delamination of the carbon from the substrate during the additional prints. The adhesion of the carbon to the substrate was investigated using a peel tester (Supplementary Fig. 6). Even at slow peeling speed of $1 \mathrm{~cm} \mathrm{~min}-1$ the carbon would take damage. The poor yield therefore likely occurs due to the frame sticking to the samples during the printing procedure and "snapping off". We further investigated whether a gentler hand-pulled lacquer print would result in less delamination, and this proved to be the case with $100 \%$ yield when performed on squares of size $15 \times 15 \mathrm{~mm}$. 
a)

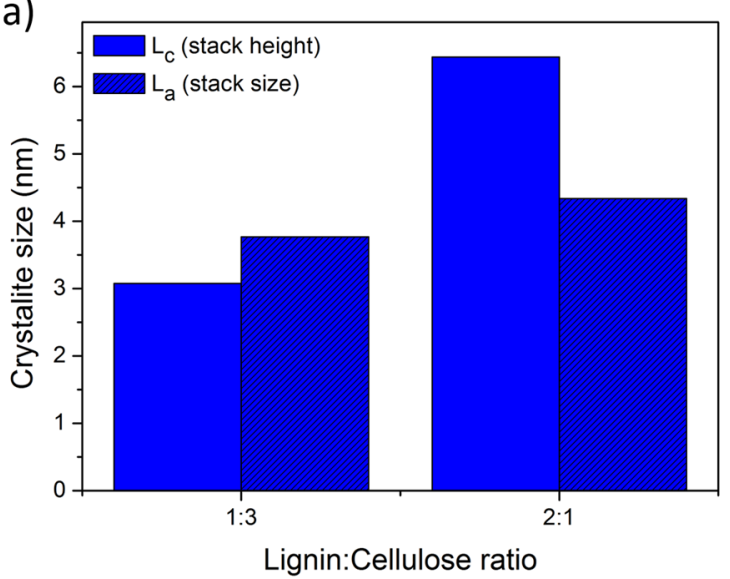

C)

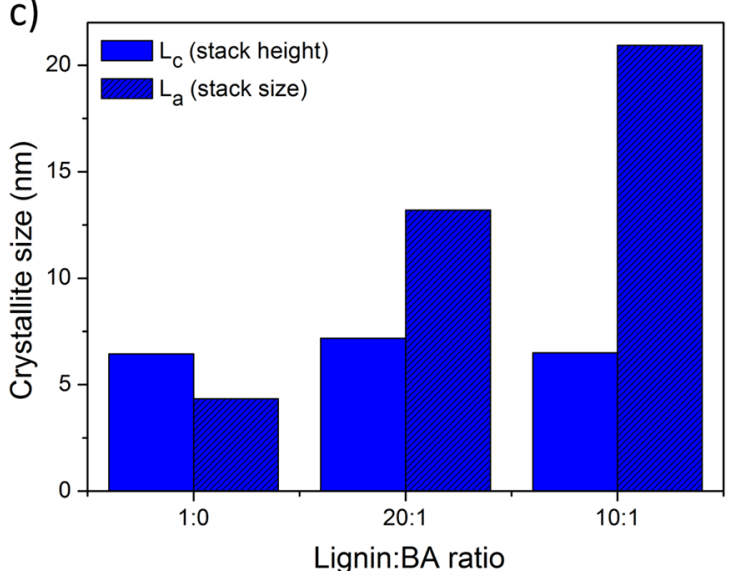

b)

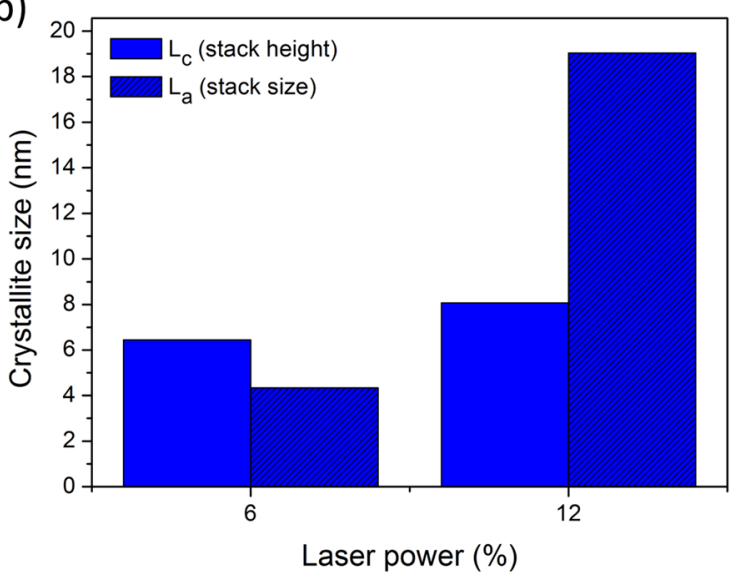

d)

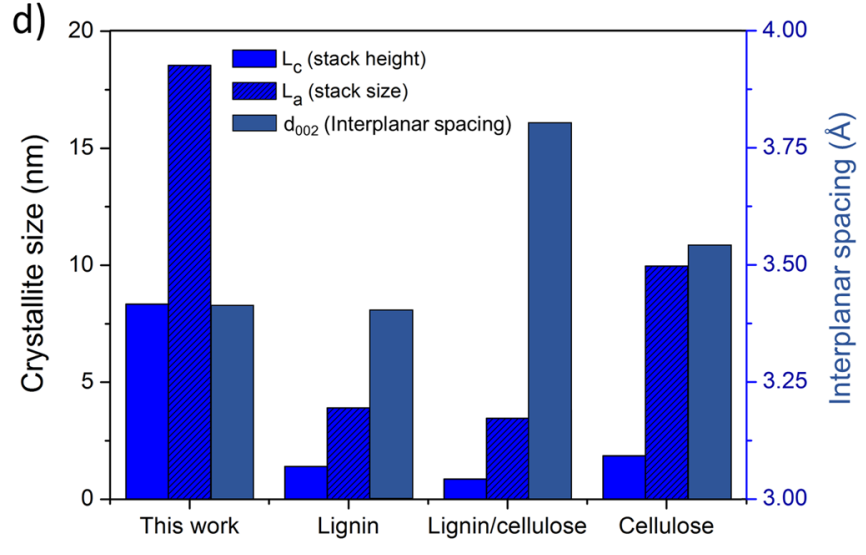

Fig. 3 Crystallite sizes derived from WAXS crystallography measurements. a Different lignin:cellulose $(\mathrm{L}: \mathrm{C})$ ratios at $6 \%$ laser power, b different laser power at 2:1 L:C ratio, c different lignin:boric acid (lignin:BA) ratio at $6 \%$ laser power and 2:1 L:C ratio, $\mathbf{d}$ comparison between carbon from this work (12\% laser power, 2:1 L:C ratio, 5:1 C:BA ratio) with literature using lignocellulosic sources graphitized in furnace ${ }^{34,41,44}$.

Finally, we performed adhesion tests of the carbon samples with protective lacquer as well as carbon resistors with silver and lacquer prints. These samples could handle peeling speeds of 50 and $100 \mathrm{~cm} \mathrm{~min}^{-1}$, respectively, without any visible damage. Future work should focus on improving the adhesion between the lignographic carbon and the substrate. However, we have shown that the carbon can be used in printed devices if a protective layer is used. The processing parameters to reduce the "snap-off" force should also be further optimized.

A key feature of printed electronics is the possibility manufacturing devices on flexible substrates. However, the devices themselves must also be able to handle bending of the substrate without damage and with stable performance over time. To test the lignographic resistors during bending, we measured the resistance of a device while wrapping it around a steel rod, giving it a bending radius of $5 \mathrm{~mm}$. Supplementary Fig. 7 shows photographs of the procedure as well as the resistance vs. bending cycle. The resistance was observed to increase by $\sim 25 \%$ during the first few cycles, but then stabilized and remained fairly constant over 100 cycles.

Humidity sensor using lignin as transducer and sensing material Up until now, we have argued for the benefit of pre-patterning the wood ink in order to avoid residual ink between carbon patterns. However, there are situations when the ink itself can be used for electronics functionality. Humidity sensors produced by printed electronics have previously been reported and typically involve printing interdigitated electrodes followed by printing a sensing layer on top of the electrodes ${ }^{45-47}$. Changes in the resistance or capacitance of the sensing layer is subsequently measured while varying the surrounding relative humidity. Here we demonstrate a humidity sensor manufactured by first patterning the ink followed by a partial graphitization to obtain interdigitated electrodes (Fig. 6a). However, the ink between the carbon electrodes is left and functions as the water-absorbing material. This method therefore involves only a single printed layer and material. Figure $6 \mathrm{~b}$ shows photographs of prints with and without graphitized electrodes, and also shows that the devices are flexible. Sensors were produced with $500 \mu \mathrm{m}$ width and separation of the finger electrodes and using two different inks with different L:C ratios. They were subsequently characterized using impedance spectroscopy at relative humidity levels between 20 and $90 \% \mathrm{RH}$ in a climate chamber (Fig. 6c, d). The obtained impedance values at $10 \mathrm{~Hz}$ was plotted vs. the humidity in Fig. $6 \mathrm{e}$. Both sensors show excellent sensitivity with an impedance spanning 5 orders of magnitude over the humidity range. It was expected that the sensor with larger amount of cellulose in the ink would have the lower impedance due to the hygroscopic nature of cellulose and therefore a larger water absorption. Instead, the sensor with high-lignin content displayed lower impedance (higher conductance). This is most likely due to the sulfonic groups on the lignin which adds ionic conductivity to the ink. Even though the sensitivity of the two devices were comparable, the lower overall impedance of the high-lignin content sensor might be preferred since it puts less demands on the input impedance of the measurement equipment. 

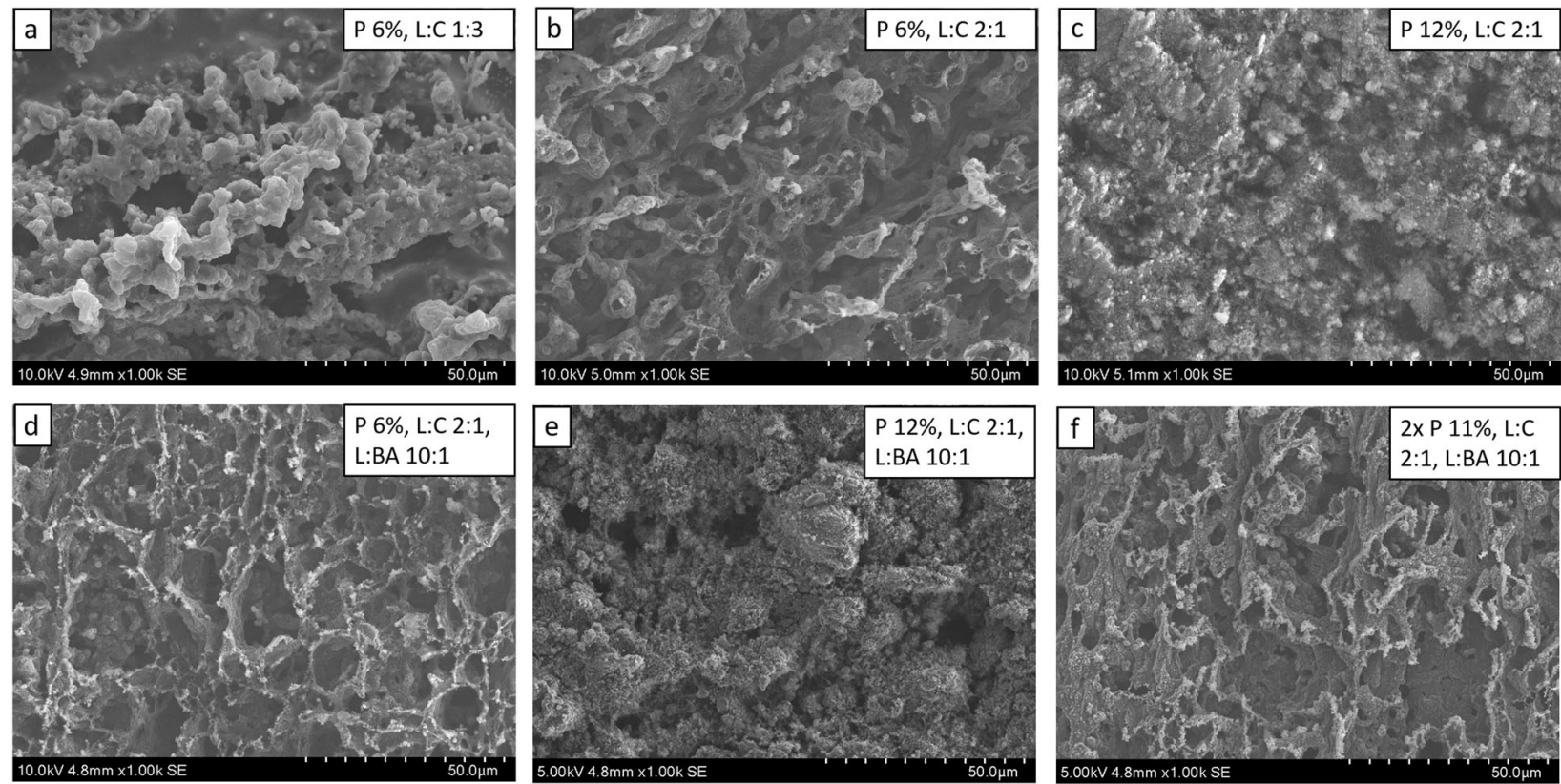

Fig. 4 SEM images of carbons produced from different ink compositions and laser parameters. The composition and laser power used to produce each sample are stated in each of the images (a-f). Scale bar $50 \mu \mathrm{m}$.

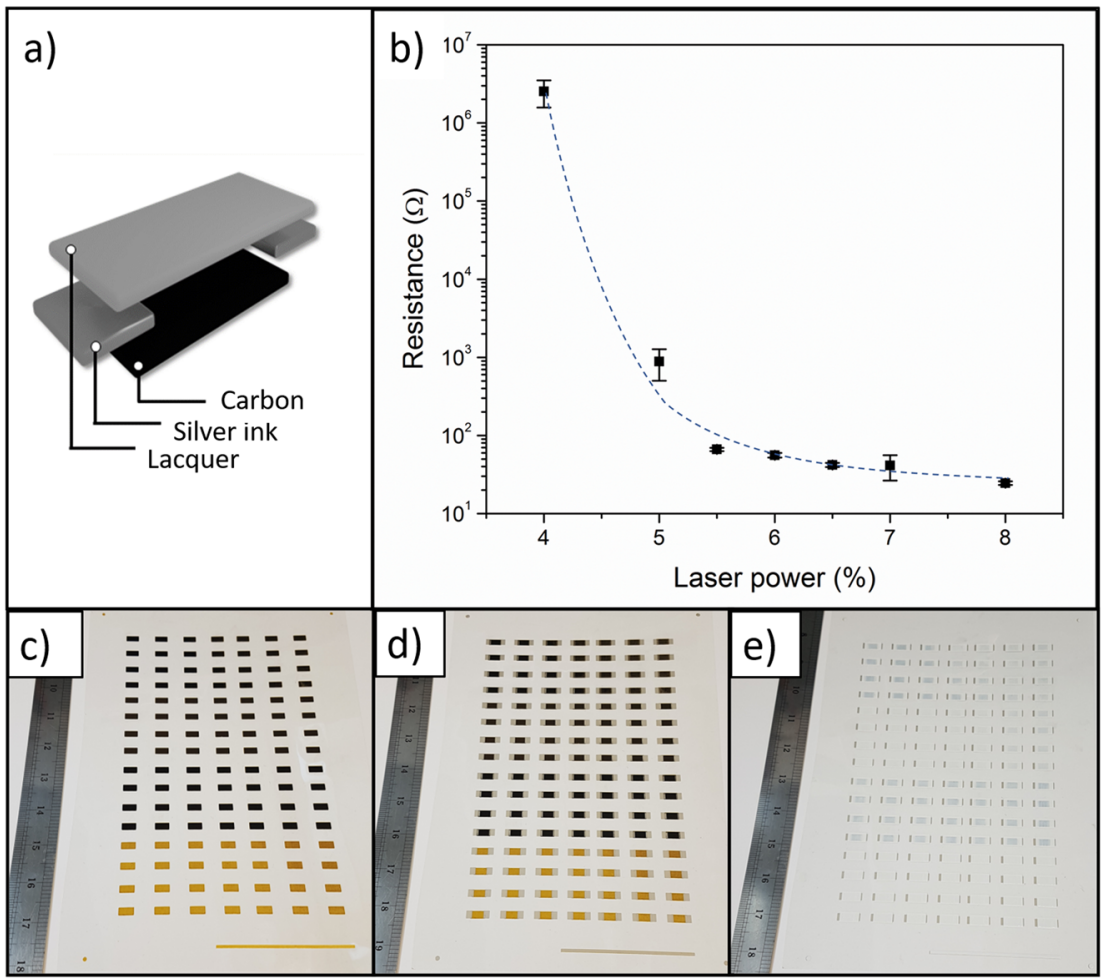

Fig. 5 Lignographic resistors using multiple printed materials. a Schematic of the resistor structure with graphitized lignin, silver contacts, and protective lacquer layer. b Resistance of the printed resistors. c Photograph of the printed lignin ink where the top part has been graphitized, $\mathbf{d}$ after the silver print, and $\mathbf{e}$ after the lacquer print (white color). The dashed line in $\mathbf{b}$ is meant as a guide for the eye.

In conclusion, we have demonstrated laser-induced graphitization on a screen printable forest-based ink obtaining record low values of sheet resistance and excellent conductivity. The prepatterning of the wood ink not only simplifies the manufacturing process, but also makes it compatible with existing printed electronics manufacturing routes. Investigations of the carbon microstructure reveals a high degree of graphitization and large crystallites, which could be obtained even at low power and in ambient atmosphere. Future work on reducing the porosity of the obtained carbon could lead to further improvement in the conductivity. We also demonstrated how the technique can be used to modulate the resistance in resistor networks by the laser 
a)

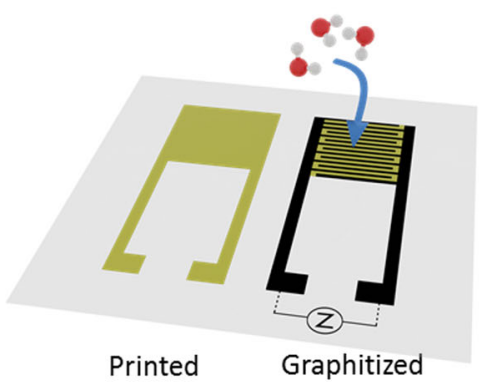

c)

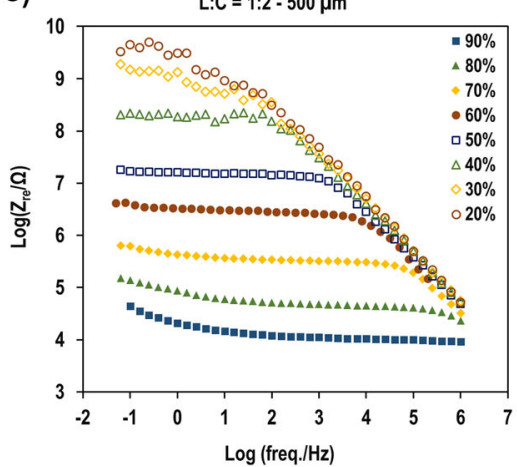

b)
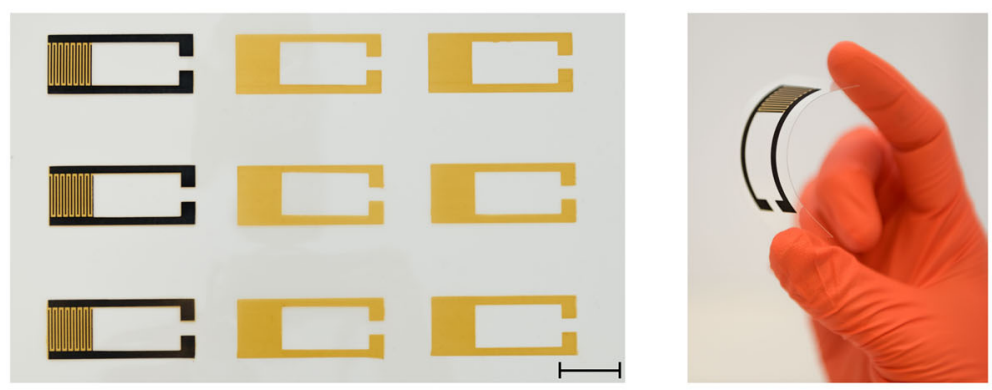

d) $\mathrm{L}: \mathrm{C}=2: 1-500 \mu \mathrm{m}$

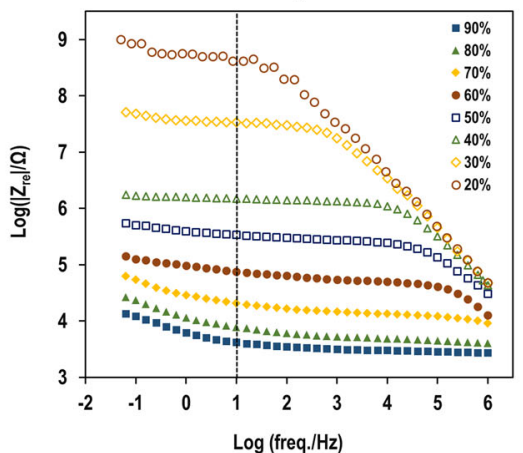

e)

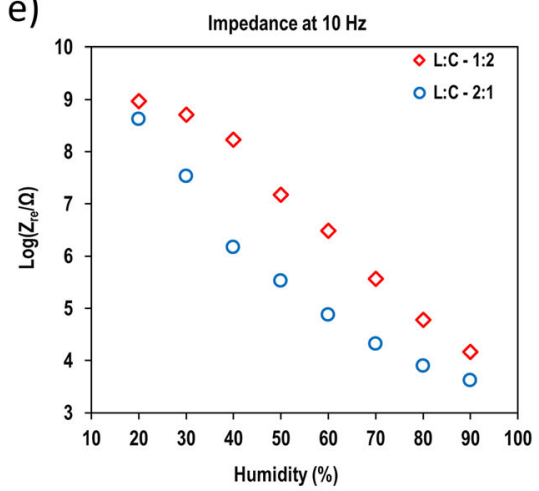

Fig. 6 Production and characterization of lignographic humidity sensors. a Schematic of the humidity sensors made with the wood ink between interdigital electrodes and using different ink compositions ( $L: C$ 1:2 and L:C 2:1). b Photographs of lignin prints and graphitized humidity sensors on flexible PET sheets. Scale bar $2 \mathrm{~cm}$. c, d The real part impedance spectra of the L:C 1:2 and L:C 2:1 device, respectively, for different relative humidity levels. e Real part impedance data from $\mathbf{b}$ and $\mathbf{c}$ taken at $10 \mathrm{~Hz}$ (as shown by the dotted lines).

power, and finally showed how the wood ink itself can be used in devices such as humidity sensors with excellent sensitivity. Although the technique was mainly demonstrated on plastic substrates, we also showed that forest/bio-based substrates like cardboard can be used. By using forest-based materials, lignography brings printed electronics one step closer to be a green and sustainable technology.

\section{METHODS}

\section{Materials}

2-Hydroxyethyl cellulose (2-HEC, $M_{\mathrm{W}}$ 90,000) DS 1.3, lignosulfonate (lignosulfonic acid sodium salt $M_{\mathrm{W}} 52,000$ ) and boric acid was purchased from Sigma Aldrich. Screen-printing frames were purchased from Coated Screens. Commercial silver ink (CXT 0644, Sun Chemical) and UV-curable lacquer (Ultra Graph UVAR 170, Marabu) were employed for printed electronics integration.

\section{Preparation of ink and printing}

The screen-printing ink was prepared by mixing $40 \mathrm{~g}$ water with $5 \mathrm{~g}$ 2-HEC and different amounts of lignosulfonate. The water was first heated on a hotplate set to $100^{\circ} \mathrm{C}$. Lignosulfonate was then slowly added to the water while stirring with a magnet bar. When the lignosulfonate was fully dissolved the 2-HEC was slowly added.

The printing procedure was performed using an E2 EKRA screen-printing machine (Supplementary Fig. 8). 120-34 screen meshes were employed for all printing processes. This corresponds to 120 threads per inch and $34-\mu \mathrm{m}$ thread diameter. Two or three layers of the ink was printed on PET substrates and baked in an oven at $80^{\circ} \mathrm{C}$ for 5 min to obtain a thickness of 8-15 $\mu \mathrm{m}$.

Integration of the lignographic materials into printed electronics was achieved by printing two layers of silver conductive tracks on the graphitized ink using screen printing (120-34 screen meshes). Followed by three layers of lacquer designed to protect the carbon and work as an electrical insulator. The silver contacts were then probed to investigate the electrical resistance of the printed stack.
Laser scribing and characterizations

The laser processing was performed on a Speedy 300 laser engraver (Trotec). The laser had a maximum power of $30 \mathrm{~W}$, maximum speed of $3.55 \mathrm{~m} \mathrm{~s}^{-1}$. The $10.6 \mu \mathrm{m} \mathrm{CO}$ laser was used for the graphitization.

For the electrical characterization, squares of size $15 \times 15 \mathrm{~mm}$ were lased on global prints of the wood ink (Fig. 2a) while varying different laser parameters such as power, speed, and $z$-offset (defocus). The sheet resistance of the squares was measured using an Ossila four-point probe system.

Impedance Spectroscopy of the humidity sensor was carried out in the frequency range of $1 \mathrm{MHz}-50 \mathrm{mHz}$ with an $\mathrm{AC}$ amplitude of $10 \mathrm{mV}$ with a computer-controlled potentiostat (SP200, BioLogic) while controlling the humidity using a climate chamber (vötschtechnik LabEvent).

Wide-angle X-ray scattering (WAXS) studies on the laser scribed samples were carried out using an Anton Paar SAXSpoint 2.0 WAXS/SAXS instrument equipped with a Dectris Inside 2D EIGER R HPC detector. The X-ray source was a microfocus $\mathrm{Cu}$ tube with point focus $\left(\lambda \sim 1.54 \mathrm{~A}^{\circ}\right)$ operated at $50 \mathrm{kV}$ and $1 \mathrm{~mA}$. The interplanar spacing of the graphene layer planes $(d)$ in both (002) and (100) crystal lattice structures were calculated using Bragg's law:

$d=\frac{n \lambda}{2 \sin \theta}$

where, $\lambda$ is the wavelength $\left(1.54 \mathrm{~A}^{\circ}\right)$, order of reflection $(n)$ is 1 , and $\theta$ is the Bragg angle of (002) or (100) reflections.

The crystallite sizes $(L)$, as out-of-plane $\left(L_{c}\right)$, and in-plane $\left(L_{a}\right)$ were calculated using Debye-Scherrer equation from characteristics of the (002) and (100) peaks, respectively.

$L=\frac{K \lambda}{\beta \cos \theta}$,

where, $\lambda$ is the wavelength, $\theta$ is the Bragg angle related to the (002) or (100) reflections, and $\beta$ is the full width at half maximum intensity (FWHM) of the (002) or (100) peaks, and $K$ is a constant taken to be 0.89 for the (002) diffraction peak and 1.84 for the (100) diffraction peak ${ }^{34}$. 


\section{DATA AVAILABILITY}

The data that support the findings of this study are available from the authors on reasonable request. The authors declare that the data supporting the findings of this study are available within the article and the corresponding Supplementary Information File.

Received: 30 January 2020; Accepted: 8 June 2020;

Published online: 06 August 2020

\section{REFERENCES}

1. Kromoser, B., Preinstorfer, P. \& Kollegger, J. Building lightweight structures with carbon-fiber-reinforced polymer-reinforced ultra-high-performance concrete: research approach, construction materials, and conceptual design of three building components. Struct. Concr. 20, 730-744 (2019).

2. Hills, G. et al. Modern microprocessor built from complementary carbon nanotube transistors. Nature 572, 595-602 (2019).

3. Aprojanz, J. et al. Ballistic tracks in graphene nanoribbons. Nat. Commun. 9, 4426 (2018).

4. Tsoukleri, G. et al. Subjecting a graphene monolayer to tension and compression. Small 5, 2397-2402 (2009).

5. Flandrois, S. \& Simon, B. Carbon materials for lithium-ion rechargeable batteries. Carbon 37, 165-180 (1999).

6. Lee, S. W., Gallant, B. M., Byon, H. R., Hammond, P. T. \& Shao-Horn, Y. “Nanostructured carbon-based electrodes: bridging the gap between thin-film lithiumion batteries and electrochemical capacitors. Energy Environ. Sci. 4, 1972-1985 (2011).

7. Wang, J. \& Sun, X. Understanding and recent development of carbon coating on LiFePO 4 cathode materials for lithium-ion batteries. Energy Environ. Sci. 5, 5163-5185 (2012)

8. Fang, R. et al. The regulating role of carbon nanotubes and graphene in lithiumion and lithium-sulfur batteries. Adv. Mater. 31, 1800863 (2019).

9. Pandolfo, A. G. \& Hollenkamp, A. F. Carbon properties and their role in supercapacitors. J. Power Sources 157, 11-27 (2006).

10. Li, Z. et al. Exceptional supercapacitor performance from optimized oxidation of graphene-oxide. Energy Storage Mater. 17, 12-21 (2019).

11. Al-Saedi, S. I., Haider, A. J., Naje, A. N. \& Bassil, N. Improvement of Li-ion batteries energy storage by graphene additive. Energy Rep. 6, 64-71 (2019).

12. Andersson Ersman, P. et al. All-printed large-scale integrated circuits based on organic electrochemical transistors. Nat. Commun. 10, 5053 (2019).

13. Brooke, R. et al. Supercapacitors on demand: all-printed energy storage devices with adaptable design. Flex. Print. Electron. 4, 015006 (2019).

14. Li, Q. et al. Review of printed electrodes for flexible devices. Front. Mater. 5, 77 (2019).

15. Lin, J. et al. Laser-induced porous graphene films from commercial polymers. Nat Commun. 5, 5714 (2014).

16. Peng, Z., Lin, J., Ye, R., Samuel, E. L. G. \& Tour, J. M. Flexible and stackable laser induced graphene supercapacitors. ACS Appl. Mater. Interfaces 7, 3414-3419 (2015).

17. Peng, Z. et al. Flexible boron-doped laser-induced graphene microsupercapacitors. ACS Nano 9, 5868-5875 (2015).

18. El-Kady, M. F., Strong, V., Dubin, S. \& Kaner, R. B. Laser scribing of highperformance and flexible graphene-based electrochemical capacitors. Science 335, 1326-1330 (2012)

19. Li, L. et al. High-performance pseudocapacitive microsupercapacitors from laserinduced graphene. Adv. Mater. 28, 838-845 (2016).

20. Nayak, P. et al. Monolithic laser scribed graphene scaffolds with atomic layer deposited platinum for the hydrogen evolution reaction. J. Mater. Chem. A 5, 20422-20427 (2017).

21. Singh, S. P. et al. Laser-induced graphene layers and electrodes prevents microbial fouling and exerts antimicrobial action. ACS Appl. Mater. Interfaces 9 18238-18247 (2017).

22. Nayak, P., Kurra, N., Xia, C. \& Alshareef, H. N. Highly efficient laser scribed graphene electrodes for on-chip electrochemical sensing applications. Adv. Electron. Mater. 2, 1600185 (2016).

23. Dou, X. et al. Hard carbons for sodium-ion batteries: structure, analysis, sustainability, and electrochemistry. Mater. Today 23, 87-104 (2019).

24. Ye, R. et al. Laser-induced graphene formation on wood. Adv. Mater. 29, 1702211 (2017)

25. Chyan, Y. et al. Laser-induced graphene by multiple lasing: toward electronics on cloth, paper, and food. ACS Nano 12, 2176-2183 (2018).
26. Zhang, W. et al. Lignin laser lithography: a direct-write method for fabricating 3D graphene electrodes for microsupercapacitors. Adv. Energy Mater. 8, 1801840 (2018).

27. White, R. H. Effect of lignin content and extractives on the higher heating value of wood. Wood Fiber Sci. 19, 446-452 (1987).

28. Khan, Y. et al. A new frontier of printed electronics: flexible hybrid electronics. Adv. Mater. 32, 1905279 (2020).

29. Linghu, C., Zhang, S., Wang, C. \& Song, J. Transfer printing techniques for flexible and stretchable inorganic electronics. npj Flex. Electron. 2, 26 (2018).

30. Zhou, H. et al. Transfer printing and its applications in flexible electronic devices. Nanomaterials. 9, 283 (2019).

31. Wicklein, B., Kocjan, D., Carosio, F., Camino, G. \& Bergström, L. Tuning the nanocellulose-borate interaction to achieve highly flame retardant hybrid materials. Chem. Mater. 28, 1985-1989 (2016).

32. Reynolds, J. A. \& Hough, J. M. Formulae for dielectric constant of mixtures. Proc. Phys. Soc., B 70, 769-775 (1957).

33. Zhao, C., Shi, Y., Xie, J., Lei, F. \& Zhang, L. Direct measurement of electrical conductivity of porous biochar monolith for supercapacitors. Mater. Res. Express. 6, 095526 (2019)

34. Shao, Y., Guizani, C., Grosseau, P., Chaussy, D. \& Beneventi, D. Biocarbons from microfibrillated cellulose/lignosulfonate precursors: a study of electrical conductivity development during slow pyrolysis. Carbon 129, 357-366 (2018).

35. Tran, T. S., Dutta, N. K. \& Choudhury, N. R. Graphene inks for printed flexible electronics: graphene dispersions, ink formulations, printing techniques and applications. Adv. Colloid Interface Sci. 261, 41-61 (2018).

36. Kim, T. H. et al. Enlarging the d-spacing of graphite and polarizing its surface charge for driving lithium ions fast. J. Mater. Chem. A 2, 7600-7605 (2014).

37. García-Negrón, V. et al. Processing-structure-property relationships for ligninbased carbonaceous materials used in energy-storage applications. Energy Technol. 5, 1311-1321 (2017)

38. Peuvot, K., Hosseinaei, O., Tomani, P., Zenkert, D. \& Lindbergh, G. Lignin based electrospun carbon fiber anode for sodium ion batteries. J. Electrochem. Soc. 166 A1984-A1990 (2019)

39. Ma, X., Yuan, C. \& Liu, X. Mechanical, microstructure and surface characterizations of carbon fibers prepared from cellulose after liquefying and curing. Materials $\mathbf{7}$, 75-84 (2014).

40. Nowak, A. P. et al. Lignin-based carbon fibers for renewable and multifunctional lithium-ion battery electrodes. Holzforschung 72, 81-90 (2018).

41. Tenhaeff, W. E., Rios, O., More, K. \& McGuire, M. A. Highly robust lithium ion battery anodes from lignin: An abundant, renewable, and low-cost material. Adv. Funct. Mater. 24, 86-94 (2014).

42. Rodríguez-Mirasol, J., Cordero, T. \& Rodríguez, J. J. High-temperature carbons from kraft lignin. Carbon 34, 43-52 (1996).

43. Wang, $\mathrm{H}$. et al. Structural evolution of rayon-based carbon fibers induced by doping boron. RSC Adv. 4, 59150-59156 (2014).

44. Xiao, H., Lu, Y., Zhao, W. \& Qin, X. A comparison of the effect of hot stretching on microstructures and properties of polyacrylonitrile and rayon-based carbon fibers. J. Mater. Sci. 49, 5017-5029 (2014).

45. Reddy, A. S. G. et al. Fully printed flexible humidity sensor. Procedia. Eng. 25 120-123 (2011).

46. Turkani, V. S. et al. A highly sensitive printed humidity sensor based on a functionalized MWCNT/HEC composite for flexible electronics application. Nanoscale Adv. 1, 2311-2322 (2019).

47. Weremczuk, J., Tarapata, G. \& Jachowicz, R. S. The ink-jet printing humidity sorption sensor-modelling, design, technology and characterization. Meas. Sci. Technol. 23, 014003 (2011).

\section{ACKNOWLEDGEMENTS}

We would like to acknowledge funding from Vinnova for the Digital Cellulose Competence Center (DCC), Diary number 2016-05193. The work was also supported by Treesearch.se.

\section{AUTHOR CONTRIBUTIONS}

All authors contributed to the preparation of this paper. J.E. conceived the idea, performed ink formulation, graphitization experiments, and electrical measurements. R.B. performed the screen printing and produced the computer graphics. O.H. and A.F. performed the WAXS and SEM measurements. K.W. performed humidity sensor measurements and took the photograhs used in Fig. 1a and 6a. M.S. supervised the work. 


\section{COMPETING INTERESTS}

The authors declare no competing interests.

\section{ADDITIONAL INFORMATION}

Supplementary information is available for this paper at https://doi.org/10.1038/ s41528-020-0080-2.

Correspondence and requests for materials should be addressed to J.E.

Reprints and permission information is available at http://www.nature.com/ reprints

Publisher's note Springer Nature remains neutral with regard to jurisdictional claims in published maps and institutional affiliations.
Open Access This article is licensed under a Creative Commons Attribution 4.0 International License, which permits use, sharing, adaptation, distribution and reproduction in any medium or format, as long as you give appropriate credit to the original author(s) and the source, provide a link to the Creative Commons license, and indicate if changes were made. The images or other third party material in this article are included in the article's Creative Commons license, unless indicated otherwise in a credit line to the material. If material is not included in the article's Creative Commons license and your intended use is not permitted by statutory regulation or exceeds the permitted use, you will need to obtain permission directly from the copyright holder. To view a copy of this license, visit http://creativecommons. org/licenses/by/4.0/.

(c) The Author(s) 2020 